\title{
¿Explicitan los autores de la Revista Médica de Chile sus fuentes de financiamiento?
}

\author{
William Romero G a, Sofía P Salas. \\ Disclosure of financial sources \\ and conflicts of interest among \\ research articles published in \\ Revista M édica de Chile
}

Background: Financial relationships between the industry and researchers have raised concerns about the existence of conflicts of interest that could influence the scientific validity of the studies. Aim: To determine the financial sources of research articles published in the Revista Médica de Chile during a five-year period. Material and Methods: Retrospective analysis of all articles classified as "research articles", published in this journal between years 2001-2005, identifying the funding source and the existence of a declaration of conflicts of interest by the authors. Results: Two hundred seventeen out of 519 research articles published in the period (42\%) had an explicit financial source disclosed. Of these, $28 \%$ were funded by internal sources, 36\% by Fondo Nacional de Desarrollo Científico y Tecnológico and 36\% by other sources. Twenty-six studies (5\%) received funding from the industry. In only five of these, the authors explicity declared the absence of conflict of interest. Among the studies that did not disclose any financial source, one third required some funding to be carried out. Conclusions: Forty two percent of research articles published in the last five years did not specify the financial source. Those that did specify a funding source were mainly supported by non-profit agencies including university centers and governmental funds. This is in contrast with international reports that evidence an important financial support from the industry. Only a minority of the authors sponsored by the industry declared absence of conflict of interest (Rev Méd Chile 2007; 135: 473-9).

(Key words: Conflict of interest; Practice guidelines; Research support)

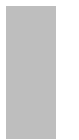

\footnotetext{
Recibido el 9 de junio, 2006. Aceptado el 12 de octubre, 2006.

Departamento de Obstetricia y Ginecología y Centro de Investigaciones Médicas, Facultad de Medicina, Pontificia Universidad Católica de Chile.

aBecario de Dermatología, Pontificia Universidad Católica de Chile.
}

$\mathrm{H}$ asta hace poco tiempo, el diseño y ejecución de la investigación biomédica era de casi exclusiva responsabilidad de instituciones acadé-

Correspondencia a: Dra. Sofía Salas I. Marcoleta 391, Santiago, Chile. Fono: (562) 354-8172. Fax: (562) 632-1924. E mail: ssalas@med.puc.cl micas, mientras que el financiamiento provenía, principalmente, de fondos públicos o de instituciones sin fines de lucro. En el último tiempo, el desarrollo de investigación biomédica de gran complejidad ha aumentado los costos económicos asociados, haciendo necesaria la búsqueda de múltiples fuentes de financiamiento, incluyendo la participación de la industria privada ${ }^{1}$. En países 
desarrollados, el financiamiento de la investigación biomédica proviene fundamentalmente de la industria farmacéutica, mientras que los fondos estatales financian principalmente investigación básica y en salud pública, los que, generalmente, no producen beneficios económicos a corto plazo, y por ello suelen no ser atractivos para la industria ${ }^{2,3}$.

En este nuevo escenario, donde los privados contribuyen activamente al desarrollo de la investigación biomédica, con un importante papel en el desarrollo de nuevos fármacos y vacunas, se ha planteado el problema de su posible influencia sobre el resultado y difusión de los estudios científicos. Esto ha sido señalado por numerosos autores, que han demostrado que aquellos estudios que cuentan con financiamiento de la industria, con mayor probabilidad muestran resultados que favorecen al producto o medicamento en estudio, respecto de aquellos trabajos con metodología comparable, pero con fuentes de financiamiento independientes ${ }^{4-7}$.

El objetivo del presente estudio fue analizar las fuentes de financiamiento de los artículos de investigación publicados en la Revista Médica de Chile y explorar la interacción de fondos públicos y privados, analizando especialmente la existencia de declaración de conflictos de interés. A este respecto, es necesario señalar que a partir de enero de 2003, los editores de la Revista Médica de Chile han considerado como parte de la política editorial, incorporar en su proceso editorial una declaración de la existencia o inexistencia de conflicto de intereses, aplicable a todos los autores que envíen manuscritos u otros documentos con la intención de ser publicados ${ }^{8}$. Como objetivo adicional, nos interesó determinar si hubo trabajos que requiriéndolo, no declaraban contar con financiamiento.

\section{Material y MÉTOdos}

Se efectuó un análisis retrospectivo de todos los artículos publicados entre enero de 2001 y diciembre de 2005 como texto completo en la sección «Artículos de Investigación»de la Revista Médica de Chile en su versión electrónica ${ }^{9}$. Cabe señalar que a partir del año 2000, el Comité Editorial de la Revista decidió fusionar en una sola sección, llamada
«Artículos de Investigación», aquellos artículos que antes se publicaban bajo la modalidad de Experiencias Clínicas»con los de «rabajos de Investigación» ${ }^{0}$. Según su metodología, los trabajos fueron clasificados en: descriptivos u observacionales, casos y controles, de cohorte, experimentales y artículos de revisión. En aquellos trabajos con metodología mixta, se consultó a un especialista en bioestadística para clasificar el tipo de estudio.

Según la fuente de financiamiento, cada artículo se clasificó en:

- Sin financiamiento explícito: cuando no se encontró dicha información.

- Interna: si correspondía a un organismo o fondo de la misma institución a la que pertenecían los autores del trabajo.

- FONDECYT: si el trabajo fue financiado total o parcialmente por el Fondo Nacional de Desarrollo Científico y Tecnológico.

- Otra: si la fuente de financiamiento correspondía a instituciones externas, tanto nacionales como internacionales, con o sin fines de lucro, o laboratorios farmacéuticos.

A juicio de estos autores, los siguientes tipos de estudios requerían de algún tipo de financiamiento:

- Estudios con exámenes o procedimientos sin fines diagnósticos, pronósticos o terapéuticos, o que no corresponden a la práctica habitual según las guías de buena práctica clínica.

- Estudios de tamizaje poblacional, en los cuales se realiza una acción o procedimiento que no corresponde a lo habitual y en los cuales, por el tipo de acción, se supone que hubo un costo adicional.

- Estudios con seguimiento prospectivo, para lo cual hay costos involucrados en efectuar el seguimiento, realizar control médico, etc.

- Estudios de laboratorio o experimentales.

- Ensayos clínicos.

En esta clasificación, se excluyó de la necesidad de requerir financiamiento adicional a los estudios basados en la administración de encuestas o cuestionarios, aún cuando en ocasiones, por el alto volumen, igual es dable suponer que hubo costos asociados. También se revisó si aquellos trabajos financiados total o parcialmente por la industria declaraban o no la presencia de potenciales conflictos de interés. 
En forma adicional, revisamos si artículos publicados en las secciones Cartas al Editor, Artículos de Revisión, Casos Clínicos, y Artículos Especiales podían constituir potenciales fuentes de conflictos de interés al promover el uso de determinados medicamentos o insumos, o si contenían normas terapéuticas que pudiesen estar influenciadas por la industria farmacéutica.

Los dos autores extrajeron los datos en forma independiente y las discrepancias fueron discutidas hasta alcanzar consenso.

\section{Resultados}

Durante los años 2001 a 2005, la Revista Médica de Chile publicó 519 artículos en la sección «Artículos de Investigación». De éstos, 360 (69,6\%) correspondieron a estudios descriptivos u observacionales, $79(15,0 \%)$ a estudios de casos y controles, $44(8,0 \%)$ a estudios de cohorte, 34 $(7,0 \%)$ a estudios experimentales y $2(0,4 \%)$ a revisiones (Figura 1).

Con respecto a las fuentes de financiamiento, ésta se explicitó sólo en 217/519 (42\%) de los artículos, mientras que en 302 no hubo referencia al financiamiento. Se observó que cerca de $60 \%$ de los estudios experimentales explicitaba fuente de financiamiento, lo que disminuía a menos de la mitad en los otros tipos de estudios: casos y controles (49\%), estudios descriptivos (39\%) y estudios de cohorte (34\%) (Tabla 1). De los 217 artículos con financiamiento explícito, 61 (28,1\%) fueron financiados por fuentes internas, 79 $(36,4 \%)$ por Fondecyt y $77(35,5 \%)$ por otras fuentes (Tabla 2). Estas fuentes fueron laborato-

Tabla 1. Análisis del financiamiento según tipo de estudio de los artículos de investigación publicados en la Revista M édica de Chile, años 2001-2005.

\begin{tabular}{|lrr|}
\hline Tipo de estudio & \multicolumn{3}{c|}{ Financiamiento explícito } \\
& No & \multicolumn{1}{c|}{$\mathrm{Si}$} \\
\hline Descriptivos/observacionales & 218 & 142 \\
Casos-Controles & 40 & 39 \\
Cohorte & 29 & 15 \\
Experimentales & 14 & 20 \\
Revisiones & 1 & 1 \\
Total n (\%) & $302(58)$ & $217(42)$ \\
\hline
\end{tabular}

\section{Tabla 2. Fuente de financiamiento de los artículos de investigación publicados en la Revista M édica de Chile, años 2001-2005, según tipo de estudio}

\begin{tabular}{|lccc|}
\hline Tipo de estudio & \multicolumn{3}{c|}{ Fuente financiamiento } \\
& Interna & FONDECYT & Otra \\
\hline Descriptivos & 43 & 46 & 53 \\
Casos-Controles & 10 & 20 & 9 \\
Cohorte & 3 & 7 & 5 \\
Experimentales & 5 & 6 & 9 \\
Revisiones & & & 1 \\
Total n (\%) & $61(28)$ & $79(36,4)$ & $77(35,6)$ \\
\hline
\end{tabular}

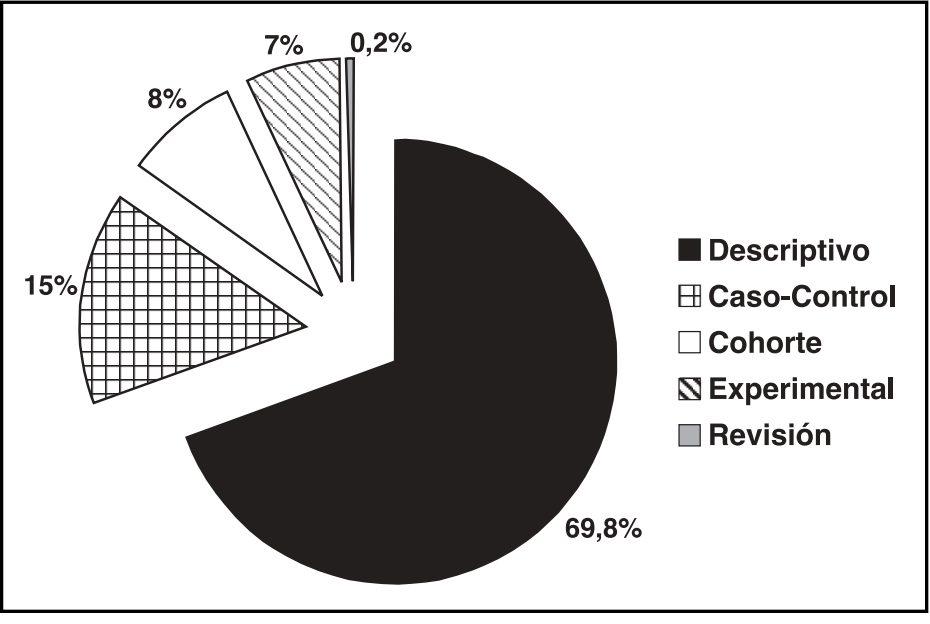

Figura 1. Análisis del tipo de estudios publicados por la Revista Médica de Chile en la sección «Artículos de Investigación»entre enero de 2001 y diciembre de 2005 . 
rios farmacéuticos o empresas privadas ( $\mathrm{n}=26,5 \%$ del total de artículos), organismos nacionales sin fines de lucro $(\mathrm{n}=26)$, tales como Gobiernos Regionales y Ministeriales, municipalidades, y organizaciones comunitarias; y organismos internacionales sin fines de lucro $(\mathrm{n}=25)$, tales como Wellcome Trust, National Institute of Health (NIH), Gobierno de Canadá y Gobierno de México, entre otras.

Según los criterios definidos, estimamos que 105 de los 302 (35\%) artículos sin financiamiento explícito, deberían haberlo tenido. A este respecto, no registraban financiamiento 61 estudios basados en exámenes o procedimientos sin fines diagnósticos, pronósticos o terapéuticos; 10 estudios de tamizaje poblacional; 19 estudios de seguimiento prospectivo; 9 estudios de laboratorio o experimentales y 6 ensayos clínicos.

Sólo cinco de los veintiséis artículos financiados por la industria declararon no tener conflictos de intereses y todos fueron publicados en fecha posterior al momento en que se introdujo la \eclaración Conflictos de Interés» como requisito para la publicación. Los restantes 21 artículos no señalaron en forma explícita la participación de las fuentes de financiamiento en el diseño, desarrollo, análisis o publicación del estudio.

Durante este período, se publicaron 21 artículos en las otras secciones de la Revista Médica de Chile, los que podrían constituir eventuales fuentes de conflictos de intereses si fueron patrocinados por la industria. Sólo un artículo señala a FONDECYT como fuente de financiamiento, mientras que en otro se señala que la filiación del autor es una empresa farmacéutica. Ninguno contiene declaración sobre conflictos de intereses.

\section{DisCUSIÓN}

La investigación biomédica es esencial para el desarrollo de la medicina. Sin embargo, cada vez es más costosa, por lo que requiere de fuentes de financiamiento que la sustenten. El presente trabajo muestra que la mayoría de los artículos de investigación publicados en la Revista Médica de Chile durante el último quinquenio no cuentan con financiamiento explícito, a pesar que, a juicio de estos autores, aproximadamente un tercio sí lo requerińa. Por otra parte, de los que sí lo mencio- nan, las principales fuentes de financiamiento provienen de las propias instituciones, fuentes gubernamentales y de otros entes sin fines de lucro, con escasa participación de la industria privada.

Existe consenso en el mundo científico que la falta de financiamiento produce disminución de la calidad de los trabajos y limita a nuevos investigadores, especialmente con ideas innovadoras, que se pierden por falta de recursos ${ }^{11}$. La falta de financiamiento explícito en casi $60 \%$ de los artículos de investigación publicados por la Revista Médica de Chile es un hecho interesante, dado los elevados costos que la investigación biomédica tiene en la actualidad. Esto podría explicarse debido a que la mayor parte de los estudios analizados corresponden a trabajos descriptivos, los que suelen no requerir grandes inversiones para su ejecución, aunque a nuestro juicio al menos 35\% de los artículos sin fuente de financiamiento explicitada, sí requerían financiamiento externo. Esto puede reflejar simple omisión por parte de los investigadores o representar un grave conflicto ético si se traspasaron los costos de la investigación a los propios participantes 0 a las aseguradoras de salud. Desde el punto de vista de la bioética principialista, no se estaría respetando el principio de justicia, puesto que algunos tendrían todas las cargas de la investigación, para beneficio de otros. Debemos reconocer, eso sí, que una de las limitaciones del presente trabajo es que los autores no fueron contactados para determinar si el estudio contó con financiamiento externo.

Otro aspecto interesante del presente estudio es la relativa baja participación de la industria en el financiamiento de la investigación biomédica chilena. Al analizar los estudios con fuente de financiamiento explícita, encontramos que sólo $5 \%$ declaraba haber sido financiada por la empresa privada, lo que difiere completamente de lo observado en países desarrollados. Estados Unidos de Norteamérica invierte más de $5 \%$ del presupuesto nacional de salud en investigación, a través del NIH, sin embargo, el año 2003 fue la industria privada la principal fuente de financiamiento de la investigación biomédica, patrocinando $57 \%$ de los estudios, seguida por el NIH con $28 \% 12$. Actualmente la industria farmacéutica financia cerca de $70 \%$ de todos los ensayos clínicos con fármacos en dicho país ${ }^{13}$. 
Se ha postulado que la industria podría estar influyendo en la agenda de la investigación biomédica, privilegiando proyectos que se traduzcan rápidamente en beneficios económicos, en detrimento de investigación básica o innovadora, que representa un riesgo desde el punto de vista económico ${ }^{14}$. Así, ha crecido el aporte para estudios de materiales e instrumentos médicos, los que requieren menor inversión para llevarlos a la práctica médica diaria, mientras disminuyen los estudios de nuevos fármacos, probablemente por los mayores costos asociados ${ }^{12}$, reportándose además dificultad para conseguir financiamiento para estudiar patologías de baja prevalencia o endémicas de países en desarrollo ${ }^{15}$.

Patsopoulos et $\mathrm{al}^{16}$, revisaron las fuentes de financiamiento de los artículos más citados en la literatura clínica entre los años 1994 y 2003; encontraron que $60 \%$ de estos artículos fueron financiados por fondos públicos y $36 \%$ por la industria privada. Sin embargo, observaron un incremento de los artículos financiados por la industria en los últimos años. La mayor participación de la industria se observó en los ensayos controlados randomizados, observándose que 31 de los 32 ensayos más frecuentemente citados en la literatura médica fueron financiados por fuentes privadas.

Diversos autores han demostrado que la fuente de financiamiento puede influir en los resultados y en la difusión de la investigación biomédica. Stelfox et $\mathrm{al}^{17}$ analizaron estudios sobre la seguridad de los bloqueadores de canales de calcio y encontraron que $96 \%$ de los autores que apoyaban la seguridad de estos medicamentos tenían financiamiento de laboratorios productores del fármaco, comparado con $60 \%$ de los autores neutrales. Bekelman et al ${ }^{18}$ analizaron 1.140 trabajos, publicados entre 1980 y 2002, y encontraron que las conclusiones de los trabajos auspiciados por la industria privada fueron significativamente más favorables para la intervención que los estudios independientes, y además, observaron que el auspicio de la industria se asoció con restricciones para la publicación y difusión de los resultados obtenidos. Lexchin et al, al estudiar la relación entre apoyo de la industria privada y los resultados de las investigaciones médicas, encontraron un tipo de sesgo sistemático en los estudios financiados por la industria a favor de las compa- ñías en cuestión, en comparación con estudios independientes. Este sesgo era independiente de la enfermedad o fármaco en estudio y de la metodología empleada ${ }^{19}$. Lo anterior no necesariamente puede atribuirse a una responsabilidad directa de las compañías patrocinadoras, sino que puede representar una compleja interacción entre los intereses de los autores, los patrocinadores e incluso los editores de revistas biomédicas, que con mayor probabilidad publicarán estudios con resultados positivos, generando un sesgo tácito para aquellos trabajos que no demuestran utilidad de la intervención en estudio ${ }^{8,20}$.

Estas observaciones han hecho surgir el concepto de conflicto de interés en la relación del investigador médico con su patrocinador. Un investigador está en conflicto de interés cuando tiene la obligación ética de poner primero el interés de su paciente, pero tiene un interés privado, que tiende a interferir con el apropiado ejercicio de su juicio sobre el bienestar de su paciente o su investigación. Un investigador con conflicto de interés puede influenciar indebidamente el diseño del estudio, la recolección de datos y el análisis o la difusión de los resultados, lo que obviamente limita la validez del estudio ${ }^{21}$. Para solucionar este problema, importantes revistas biomédicas como New England Journal of Medicine, Lancet, Journal of the American Medical Association y Canadian Medical Association Journal han sido pioneras en promulgar un conjunto de requerimientos éticos para sus autores, que consiste en la exigencia de una declaración de conflictos de intereses, donde se detalla la afiliación de los autores y el rol de los patrocinadores en el estudio, además de una declaración de autoría donde el autor acepta la total responsabilidad por la conducción del estudio, que tuvo acceso completo e irrestricto a todos los resultados y que fue suya la decisión de publicación ${ }^{22}$. Tal como se mencionó, la Revista Médica de Chile incorporó exigencias similares para sus autores, reconociendo la importancia de mantener la objetividad de los investigadores médicos ${ }^{8}$.

El problema de los conflictos de intereses no es un tema resuelto. La Asociación Americana de Escuelas de Medicina, en el año 2004, reportó el fortalecimiento de las normas sobre conflictos de intereses en estudios con seres humanos, aunque reconoció la falta de políticas uniformes a este 
respecto ${ }^{23}$. En nuestro estudio, encontramos que menos de $20 \%$ de los artículos que recibieron aportes de la industria privada manifestaron, en forma explícita, no tener conflictos de intereses. Esto es preocupante y creemos necesario aumentar las exigencias a los investigadores, para asegurar la integridad de la investigación médica en Chile. El presente estudio abarcó un período de tiempo que antecede a la recomendación sobre conflictos de interés efectuada por la Revista Médica de Chile ${ }^{8}$, lo que explicaría en parte la baja declaración de la presencia de conflictos de interés.

Creemos importante destacar, que a pesar de la escasa declaración de ausencia de conflictos de intereses en los trabajos financiados por la industria en la Revista Médica de Chile, sólo $5 \%$ de los artículos analizados fueron financiados con fondos privados, mientras que más de $90 \%$ de los artículos que declararon el origen de sus fondos fueron financiados por organismos sin fines de lucro. Esto hace menos probable la existencia de conflictos de interés y de sesgos en los resultados publicados, otorgando mayor validez a los estudios.

Finalmente, no podemos dejar de mencionar que nuestros resultados muestran que la gran mayoría de los trabajos de investigación publicados en la principal revista médica chilena corresponden a estudios descriptivos u observacionales. Esto contrasta con los resultados de una reciente publicación que muestra que los trabajos más

\section{REFERENCIAS}

1. Cohen JJ, Siegel EK. Academic medical centers and medical research: the challenges ahead. Jama 2005; 294: 1367-72.

2. National Institutes of Health. Selected research advances of NIH. Disponible en http:// www.nih.gov/od/museum/history/advances.htm. [Consultado el 11 de abril de 2006].

3. Nathan DG, Fontanarosa PB, Wilson JD. Opportunities for medical research in the $21^{\text {st }}$ century. JAMA 2001; 285: 533-4.

4. WAHLBECK K, ADAMS C. Beyond conflict of interest. Sponsored drug trials show more-favourable outcomes. BMJ 1999; 318: 465. citados en la literatura médica internacional son, en su gran mayoría, ensayos clínicos controlados aleatoriamente y trabajos experimentales, mientras que sólo 20/289 correspondieron a trabajos descriptivos ${ }^{16}$.

En conclusión, el presente análisis muestra que la participación de la industria privada en el financiamiento de la investigación biomédica publicada en la Revista Médica de Chile en los últimos cinco años es escasa. Es posible suponer que gracias al progreso de la ciencia y la medicina en Chile, nos estemos acercando a la realidad de países desarrollados, donde la influencia de privados en la investigación biomédica es cada vez mayor. Creemos que la participación de la empresa privada es importante en el desarrollo del avance del conocimiento biomédico, como ha sido demostrado por experiencias extranjeras que muestran que los trabajos de mayor impacto tienen financiamiento privado. Sin embargo, creemos que es esencial clarificar las fuentes de financiamiento de la investigación y declarar detalladamente los posibles conflictos de interés, para asegurar la validez de nuestros estudios y nuestra integridad como investigadores. A este respecto, es conveniente señalar que también existe una responsabilidad por parte del Comité Editorial de la Revista, en el sentido de hacer cumplir las normas vigentes sobre declaración de conflictos de interés.

5. Djulbegovic B, Lacevic M, Cantor A, Fields KK, BenNetT CL, ADAMS JR ET AL. The uncertainty principle and industry-sponsored research. Lancet 2000; 356: 635-8.

6. Procyshyn RM, Chau A, Fortin P, Jenkins W. Prevalence and outcomes of pharmaceutical industry-sponsored clinical trials involving clozapine, risperidone, or olanzapine. Can J Psychiatry 2004; 49: 601-6.

7. KJAergard L, Als-Nielsen B. Association between competing interests and authors' conclusions: epidemiological study of randomised clinical trials published in the BMJ. BMJ 2002; 325: 249.

8. Reyes H, Palma J, Andresen M. La importancia de declarar un conflicto de intereses en las revistas médicas. Rev Méd Chile 2003; 131: 7-9. 
9. Múltiples autores. Revista Médica de Chile. Disponible en: http://www.scielo.cl/scielo.php?script= sci serial\&pid $=0034-9887 \& \operatorname{lng}=$ es\&nrm $=$ iso Revista Médica de Chile 2001-2005. [Consultado en enero-febrero 2006].

10. Reyes H, KaufFManN T, AndRESen M. [Research articles]. Rev Méd Chile 2000; 128: 7-8.

11. Marks AR. The economy of science. J Clin Invest 2004; 114: 871.

12. Moses $H, 3^{R D}$, Dorsey ER, Matheson DH, Thier SO. Financial anatomy of biomedical research. JAMA 2005; 294: 1333-42.

13. BodenheIMER T. Uneasy alliance-clinical investigators and the pharmaceutical industry. N Engl J Med 2000; 342: 1539-44.

14. SмITH R. Curbing the influence of the drug industry: a British view. PLoS Med 2005; 2: e241.

15. StAROPOL JF. MSJAMA: Funding and practice of biomedical research. JAMA 2003; 290: 112.

16. Patsopoulos NA, IoanNidis JP, Analatos AA. Origin and funding of the most frequently cited papers in medicine: database analysis. BMJ 2006; 332: 1061-4.

17. Steifox HT, Chua G, O’Rourke K, Detsky AS. Conflict of interest in the debate over calcium-channel antagonists. N Engl J Med 1998; 338: 101-106.
18. Bekelman JE, Li Y, Gross CP. Scope and impact of financial conflicts of interest in biomedical research: a systematic review. JAMA 2003; 289: 454-65.

19. Lexchin J, Bero LA, Djulbegovic B, Clark O. Pharmaceutical industry sponsorship and research outcome and quality: systematic review. BMJ 2003; 326: 1167-70.

20. SCHAFER A. Biomedical conflicts of interest: a defence of the sequestration thesis-learning from the cases of Nancy Olivieri and David Healy. J Med Ethics 2004; 30: 8-24.

21. THOMPson DF. Understanding financial conflicts of interest. N Engl J Med 1993; 329: 573-6.

22. InTERNATIONAL CommitTeE of Medical Journal Editors. Uniform requirements for manuscripts submitted to biomedical journals: updated October 2001. Disponible en: http://www.icmje.org/. [Consultado el 30 de marzo de 2006].

23. Ehringhaus S, Korn D. U.S. Medical School Policies on Individual Financial Conflicts of Interest. Results of an AAMC Survey. Disponible en: http:// www.aamc.org/members/coitf/coiresults2003.pdf. [Consultado el 25 de marzo de 2006].

Agradecimientos

Agradecemos en forma especial a la Srta. Alessandra Gederlini, estadística del Centro de Investigaciones Médicas de la Pontificia Universidad Católica de Chile, por su ayuda para clasificar los artículos de investigación estudiados. 